FERMILAB-Conf-90/79

\title{
Computing and Data Handling Recent Experiences at Fermilab and SLAC ${ }^{*}$
}

\author{
Peter S. Cooper \\ Fermi National Accelerator Laboratory \\ P.O. Box 500 \\ Batavia, Illinois 60510
}

April 9, 1990

* Talk presented at the 1990 Conference on Computing in High Energy Physics, Santa Fe, New Mexico, April 9-13, 1990. 


\title{
COMPUTING AND DATA HANDLING \\ RECENT EXPERIENCES AT FERMILAB AND SLAC
}

\author{
April 9, 1990 \\ Peter S. Cooper \\ Fermi National Accelerator Laboratory \\ PO Box 500, Batavia, IL 60510
}

ABSTRACT

Computing has become evermore central to the doing of high energy physics. There are now major second and third generation experiments for which the largest single cost is computing. At the same time the availability of "cheap" computing has made possible experiments which were previously considered infeasible. The result of this trend has been an explosion of computing and computing needs. I will review here the magnitude of the problem, as seen at Fermilab and SLAC, and the present methods for dealing with it. I will then undertake the dangerous assignment of projecting the needs and solutions forthcoming in the next few years at both laboratories. I will concentrate on the "offline" problem; the process of turning terabytes of data tapes into pages of physics journals.

\section{INTRODUCTION}

Computing has come a long way in high energy physics. The newly formed Computing Division at Fermilab (of which I am a member) commands $10 \%$ of the lab staff and more than $10 \%$ of the budget. Seventeen years ago we acquired our first "central" computer - as surplus from LBL! This history is well known, so I won't pursue it here. The remarkable fact remains that in a period when the cost of computing has literally dropped by orders of magnitude the fraction of available funds spent on computing and computing resources has grown substantially in high energy physics.

What I will concentrate on here is what this has lead us to. We face computing problems of enormous proportions. Furthermore, there is every reason the believe that the need for rapid growth will continue and accelerate. My topic is how we are handling these problems today and where they may take us tomorrow. I will focus on the "offline" half of the problem; the reduction and analys is of the data tapes. The scope of this paper will be my own laboratory (Fermilab) and SLAC. I wish to acknowledge and thank Chuck Dickens and Charlie Prescott of SLAC for re-educating me about SLAC's computing enterprise. The credit is theirs; the errors are mine. 
WHAT'S THE JOB?

Let's begin with an overview of the problem. Most experiments today have three or more stages, or PASSes, in their analysis. PASS1 is a reconstruction of all, or at least most, of the events on a data tape. These jobs tend to be very CPU intensive. They also have the nasty habit of making the data set larger rather than smaller. Many experiments can't (or won't) throw much away at this stage, so they add the analysed quantities to the back end of the raw data and keep it all. PASS2 is typically a recompute and sort job. The inevitable last $10 \%$ of the main event analysis which oither didn't make it into PASS1, or was done wrong and needs to be "better" gets done the second time through. Then, the various "physics streams" get sorted out in to as many as a dozen different overlapping data sets. Most of these are even smaller than the original! PASS3 is the "DST" (Data Summary Tape) stage where as many graduate students as there are on the experiment attempt to see how many tries it takes to remove the oxide from the magnetic recording material (either tape or disk) on which the DSTs have been stored. In the process they do physics, write papers and earn degrees. PASS 3 may contain the production and processing of mini, micro and even nano DSTs. Formally these would be passes 4-6 but they all tend to have the same characteristics and so I will treat them here with PASS3 as a single entity.

PASS1's are rarely done more than once. No one can stomach another yoar - and a typical PASS1 takes at least that long. PASS2's, or at least the sort phase, may happen several times as people remake their DST's with better knowledge of their constants, cuts and algorithms. PASS3's and beyond get done regularly. The students will remake the mini and micro-DSTs every day if you let them.

These PASSes have significantly different characteristics as computing jobs. PASS1s are anywhere from highly to ridiculously compute bound. They tend to need mainly logical and integer arithmetic rather than floating point calculations. Floating point is required but MIPs are more important than MegaFLOPs. PASS2's have significant compute requirenents (typically 10-20\% of PASS1) and large I/O requirements as they try to sort thousands of input tapes into dozens of hundred tape piles. PASS3's and beyond tend to do large amounts of $I / 0$; preferably from online storage. They also can be doing heavy calculations as the double precision matrices get inverted to do the complicated fits.

There are also the Monte Carlos in which people try to simulate at least there entire experiment (if not the entire world). These are completely CPU bound in and of themselves. Most groups take the sensible and prudent course of having the monte carlo write a data tape which they then feed to their PASS1-3 analysis chain re-invoking the entire monster once again. 
Finally there is everything else - software development, code management, word processing, etc. This can be look on as "infrastructure" but, in fact, it is where most of the people spend most of their computing time. If you doubt how important all this is just try to change someone's favorite system for a "better" one.

To characterize the present loads and PASS1 requirements I have chosen experiments which have already taken data at each lab. These experiments (shown in Table 1.) are of different characters and each represents, in the case of Fermilab, a class of similar experiments. I've also estimated the total lab wide load for the 1988-1990 time period. At Fermilab this included the last fixed target run in which 16 experiments took data and the last collider run in which CDF and two smaller experiments ran. At SLAC this period saw the analysis of Mark III data from SPEAR, PEP data from several detectors and Mark II data from the first runs of the SLC.

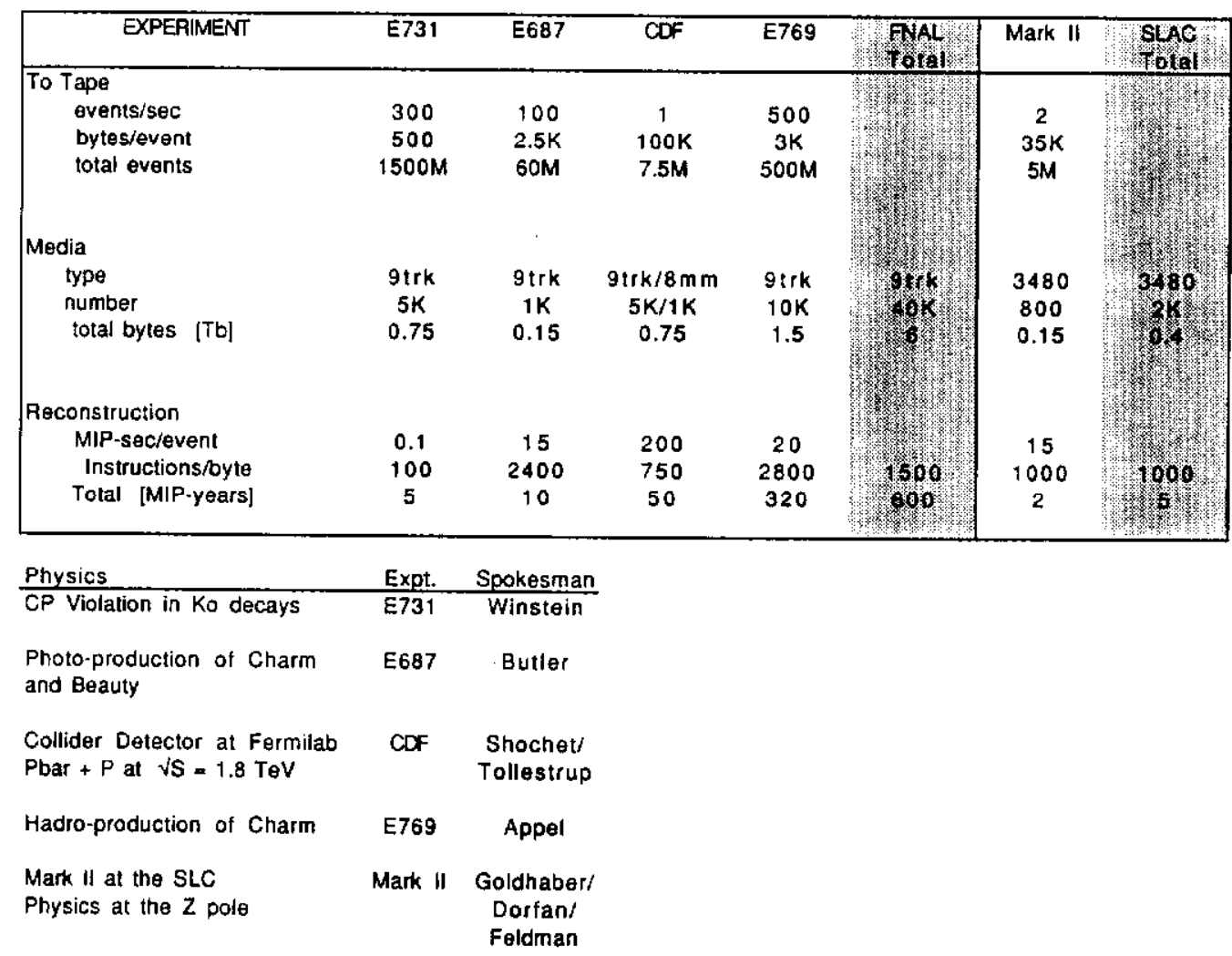

Table 1. Typical Present Experiments

The units of Table 1 require comment. I've tried to present these data in a platform and media independent manner. MIPs are in the standard units used at Fermilab ( 1 MIP $=a$ VAX 11/780). The measure of CPU boundedness is "Instructions/byte". This is the number of instructions executed per byte of data I/D (paging, swapping, constants, etc not included). Note that a job which is CPU bound on a 1 MIP VAX $11 / 780$ at 50 instructions/byte will be I/O 
bound on a $100 \mathrm{MIP}$ processor (or farm of processors). This metric is a useful way to discuss the potential performance of the same job on different platforms.

\section{HOW ARE WE DOING IT NOW?}

The presently installed central computing capacities are shown for SLAC and Fermilab in tables 2 and 3 respectively. As the table shows, SLAC has about 150 MIPs of other computing including VAXes at experiments, workstations on peoples desks, CAD/CAM systems for engineering, etc. Fermilab is proportionately larger with 500-800 such distributed MIPs. The Fermilab table contains only the "central" computing systems. It doesn't include the distributed systems for lack of space. The Fermilab reorganization of computing has recognized this reality. One of the five departments in the new computing division is "Distributed Computing" with responsibility for the systems aspects of the central VAX clusters as well as network, hardware maintenance and the other functions necessary to keep such a large and far flung computing enterprise working.

\begin{tabular}{|c|c|c|c|c|}
\hline SYSTEM., , YY & CPU & $\begin{array}{l}\text { Tape Drives } \\
9 \text { trk T } 3480 \quad 8 \mathrm{~mm}\end{array}$ & $\begin{array}{l}\text { Tape: } 1 / O \\
\mathrm{Mb} / \mathrm{sec}]\end{array}$ & Disk \\
\hline $\begin{array}{l}\text { CENTRAL COLPUTING } \\
\text { IBM Systems }\end{array}$ & 70 & 12 & 6.0 & 80 \\
\hline $\begin{array}{l}\text { IBM 3090-200E } \\
\text { IBM 3081K } \\
\text { 2-STK Robots }\end{array}$ & $\begin{array}{l}50 \\
20\end{array}$ & 16 & 12.0 & \\
\hline $\begin{array}{l}\text { DISTRIBUTED COMPUTING } \\
\text { DEC Vaxes }\end{array}$ & 44.2 & & & 28 \\
\hline $\begin{array}{l}\text { 1. VAX } 8810 \\
\text { 2.VAX } 8800 \\
1 . V A X 8600 \\
2 . \text { VAX } 785 \\
11 . \text { VAX } 780 \\
\text { 2. VAX } 750\end{array}$ & $\begin{array}{c}5 \\
20 \\
4 \\
3 \\
11 \\
1.2\end{array}$ & & & \\
\hline $\begin{array}{c}\text { Workstations } \\
\text { 47-VS2000 } \\
\text { 3-VS } 3200 \\
\text { 5-VS31000 }\end{array}$ & $\begin{array}{l}71 \\
47 \\
15 \\
9\end{array}$ & & & \\
\hline
\end{tabular}

Table 2. SLAC Systems

SLAC's central computing is a homogenous one vendor shop; a style very consistent with their present needs. Their emphas is is on having all the data available all the time. They have concentrated heavily on robotics for tape mounting and run a "lights out" computer room operation. They currently have two STK 3480 tape robots with a total capacity of $2.4 \mathrm{~Tb}$ online and available with "seek" times of less than 20 seconds and transfer rates of $3 \mathrm{Mb} / \mathrm{sec}$.

Fermilab is a collection of four major types of computing based upon the "pawnbroker" computing model (fig 1) the basis for which can be found in the Ballam committee report (ref 1) of 1983. The Fermilab implementation of this model has two very large VAX 
clusters running VAX/VMS playing the role of the interactive front-end systems where program development, mail, word processing and the like are done. Typical loads are 400-450 users logged on in the afternoon. The numeric intensive "number cruncher" is the Amdahl 5890-600E system running VM/XA. This system has been in operation for just about two years. It is now heavily used for PASS2 and PASS3 analyses. It is mainly a batch engine with most job submissions coming from a few logged on interactive users or from the VAX clusters via a DECnet connection - as the model envisioned.

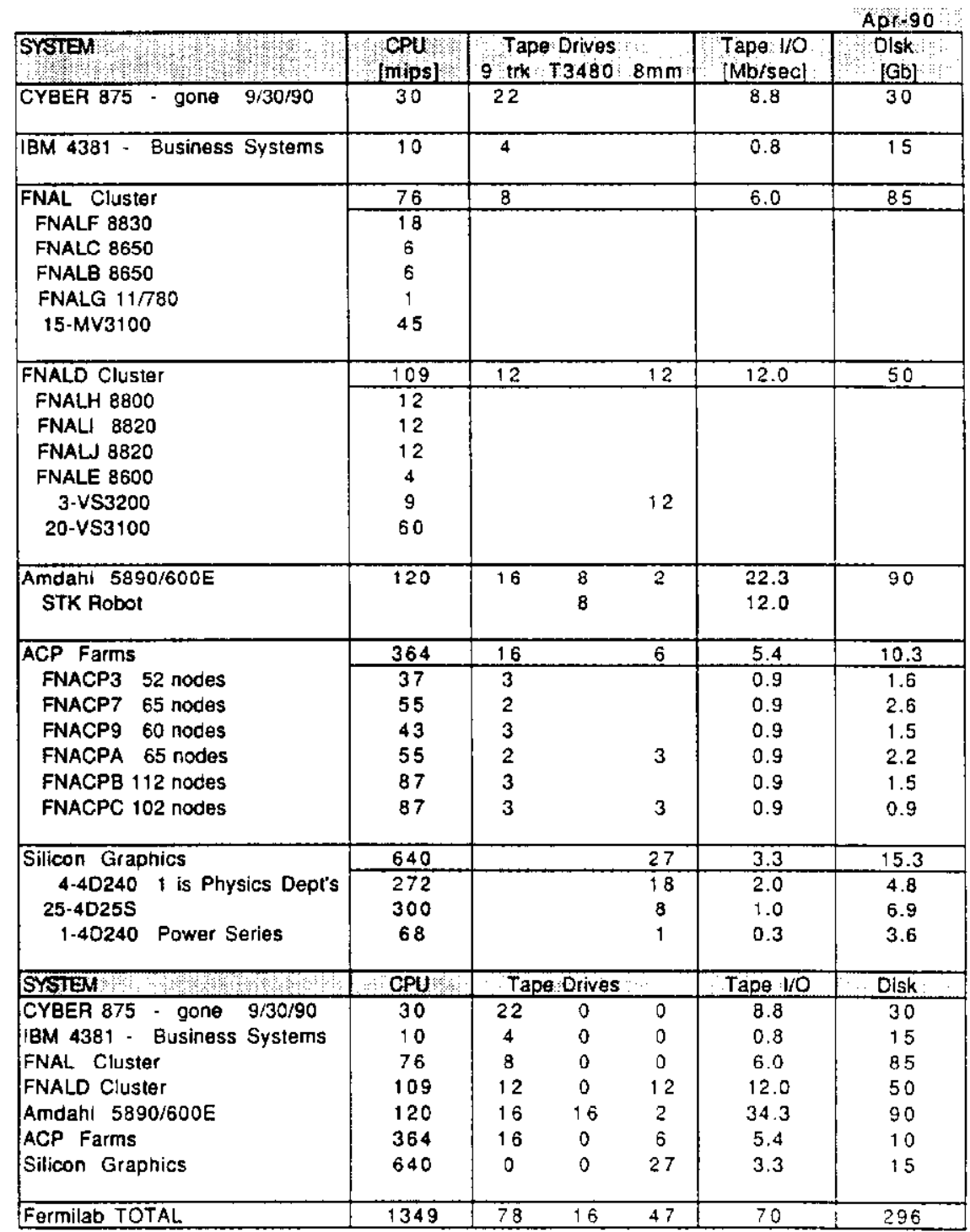

Table 3. Fermilab Systems

The third ball of the pawnbroker is "farms". These are collections of processors running "stable" computing bound production jobs (read PASS1). The processing is done in parallel with one event, or more typically one block of events, sent to each of up to 100 processors with the results of the calculation fetched 
and written back to tape. CPU powers of up to 100 MIPs are achieved with parallelism operating on a time scale of minutes rather than nanoseconds. There are two types at the moment; ACP and RISC/UNIX systems. CDF also built a farm of 20 VAXstation 3100 's to help with the completion of their PASS1. These systems have now been absorbed into one of the central VAX clusters (FNALD). The ACP farms are made of single board computers based on Motorola 68020 chips after a Fermilab design (ref 2,3). The host which controls the farm and does all the $I / 0$ is a MicroVAX 3200 . These six systems did the lions share of the PASS1 from the previous round of experiments both fixed target and collider. The RISC/UNIX boxes, presently mainly Silicon Graphics $40 / 240$ systems are in operation about 9 months. This class of computing clearly represents the "modern farm" on which the next round of experiments will do their PASS1's. The only tape $I / 0$ in use at the moment on these systems is $8 \mathrm{~mm}$. This is quite consistent with their future use at Fermilab.

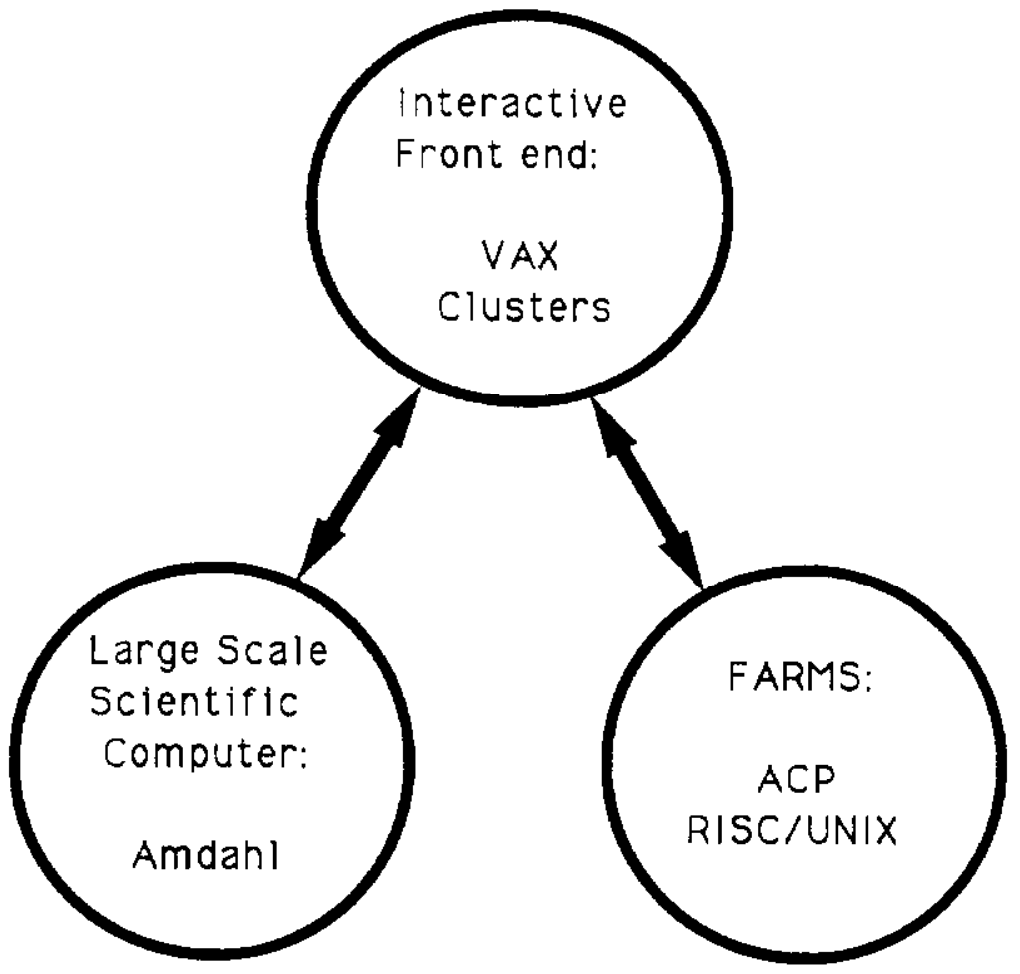

Figure 1 - The Fermilab Model of Computing

The growth profile of installed central MIPs at Fermilab is shown in figure 2. The trend is clear; in the 18 month period from the end of the previous fixed target run (April 1988) until the beginning of the new computing division (October 1989) installed MIPs doubled every six months! After a brief respite to catch our breath and reorganize we are off again on the exponential slope.

At SLAC the total load of PASS1 analysis is tractable. Electron colliders are cursed and blessed with the low event rates that the small cross-section provides. At Fermilab the total load 
is dominated by the fixed target experiments - indeed half of it is from one experiment. The cross-section is for all practical purposes infinite. The problem is how to dig out the interesting signal. Some of this is done with fast triggers and online processors which are the topics of other papers. There still remains the offline part of the job. In the case of E769 which deliberately chose to record $1 / 4$ of the inelastic cross section on tape, the rejection required to reduce their $500 \mathrm{million}$ triggers to 10,000 reconstructed charm events is of order $1 / 50,000$ or about one good event per raw 9 track data tape. The other experiments are not quite so extreme but collectively they sum to just as big a job.

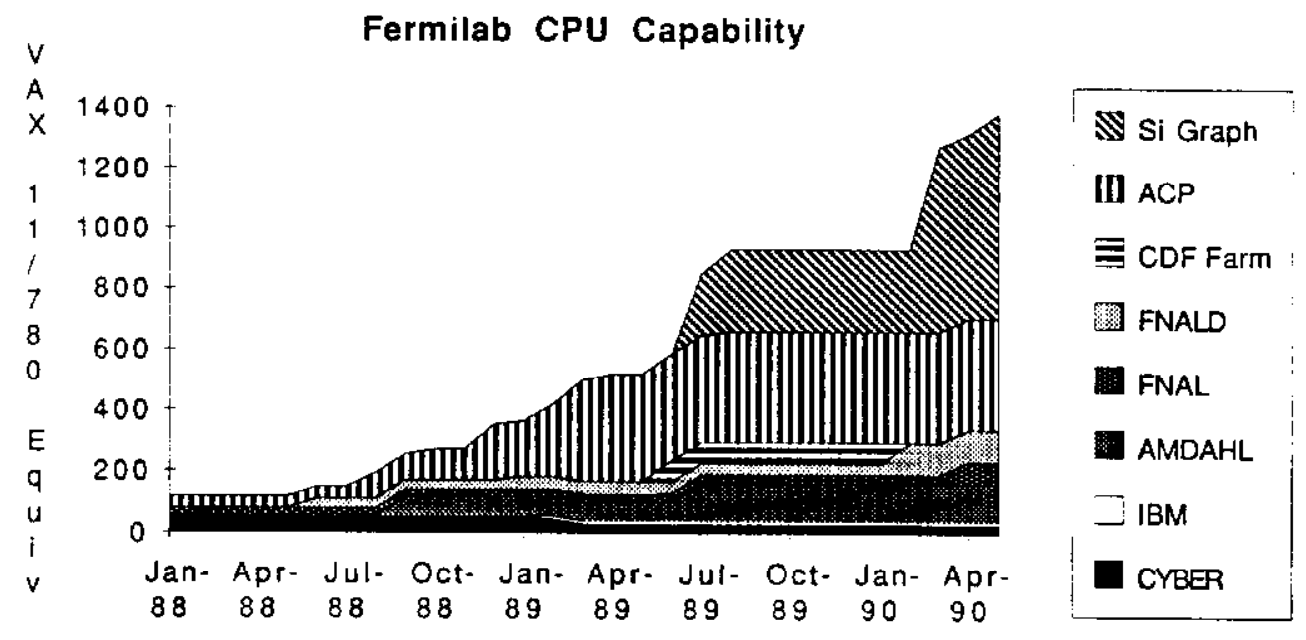

Figure 2 - Fermilab CPU Capability

The Pass2 load is an acute rather that a chronic problem. There is a substantial amount of tape handling but most experiments can get through this in a few months. A major new problem is multiple media. With 9 track, $3480 / 18$ track and $8 \mathrm{~mm}$ tapes all in active use at Fermilab presently, it always seems that the data is on the wrong medium for whatever needs to be done next.

The PASS3 loads are just seriously beginning at Fermilab from the previous data (and we have just embarked on another fixed target run in mid February). These jobs tend toward very heavy $I / 0$ usage; both tape and disk. SLAC solves this problem elegantly with their robots and homogenous system. At Fermilab with four different operating systems, three different kinds of tape media and 10 times the total data volume things are harder.

Figures 3 and 4 shown the pattern of delivered CPU and tape mounts at Fermilab over the past year. All but a few hundred of the 44,000 tape mounts last month were done by hand by operators. This activity requires a staff of 25 at a cost of just under 1 Ms per year. Our first big STK robot is scheduled for installation the week of this conference. As the growth curves clearly imply we are going to automate or die. 
Tape Mounts per Month

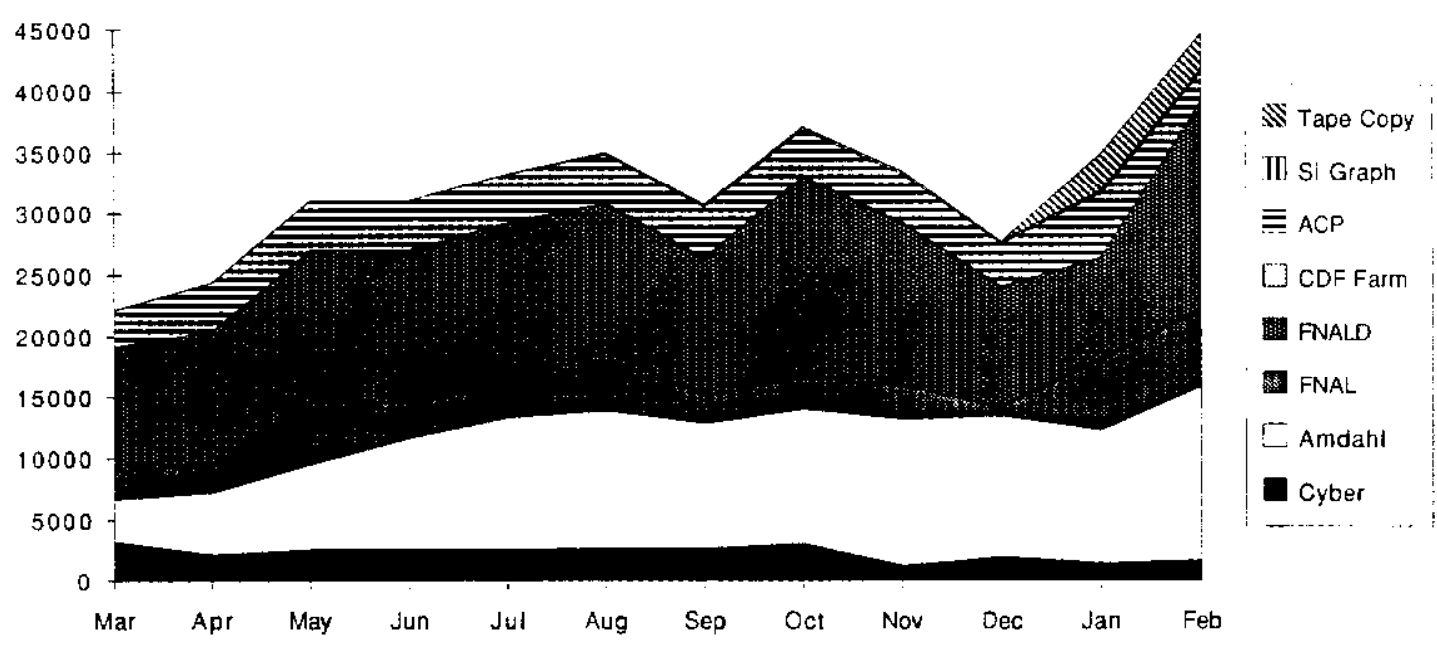

Figure 3 - Fermilab Tape usage per Month

CPU per Month

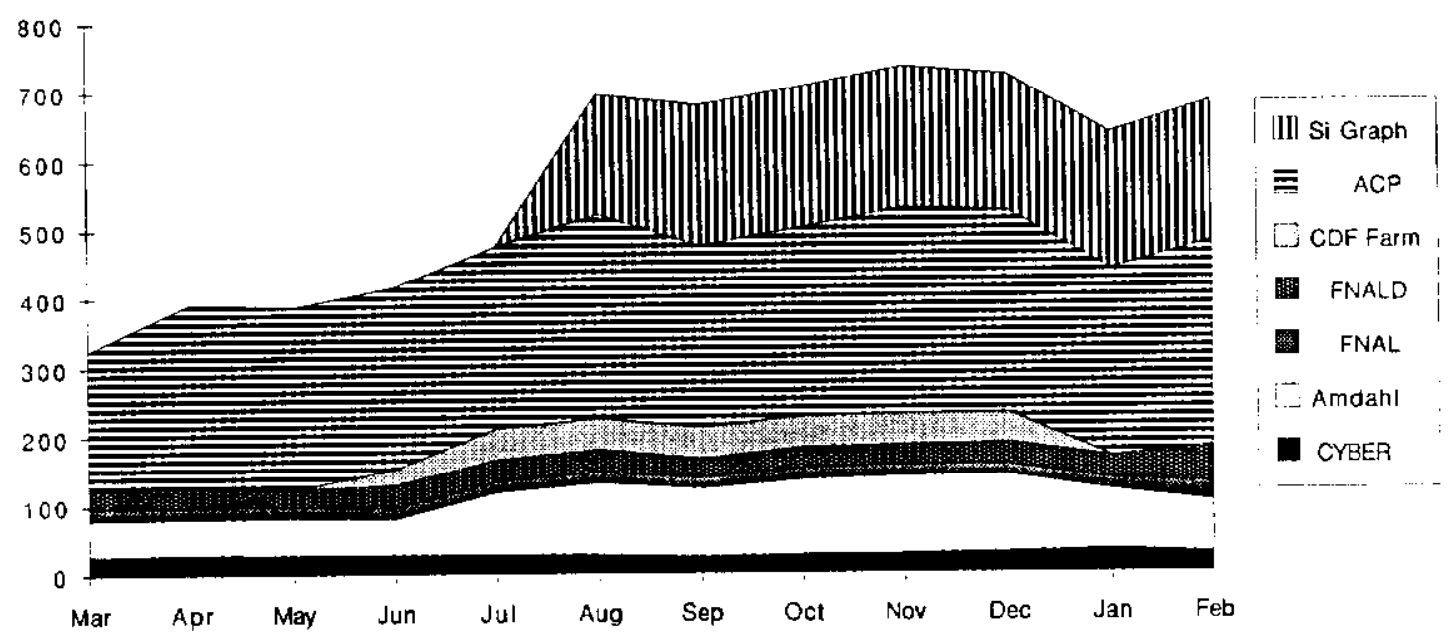

Figure 4 - Fermilab CPU usage per Month

\section{SO WHAT'S THE PROBLEM?}

In Table 4 I show a similar selection of experiments scheduled to be analysing their data in the next 2-3 years. These are analogous to those in table 1 ; in fact most are either the next run of the same experiment or the direct successor to a table 1 experiment. Data volumes are up a factor of 5-7 at both SLAC and Fermilab. The detectors are, if anything, more complicated so compute times grow somewhat. SLAC will need about a factor of two increase in CPU with proportionate growth in peripherals. At 
Fermilab we are looking at growth of a factor of 5 in total capability, from 1000 to 5000 MIPs in terms of CPU, over this three year period. (Recall that the table shows only the PASS1 requirements.) The assumption made to arrive at these factors is that an experiment should be able to complete its PASS1 in of order one year. Any longer than that and last run's data (from two years ago) won't be done before this years new data arrives.

\begin{tabular}{|c|c|c|c|c|c|c|c|}
\hline EXPERIMENT & E761 & E687 & COF & E791 & W. FAL & SLD & $\begin{array}{l}\text { SLAC } \\
\text { Solal }\end{array}$ \\
\hline \multicolumn{8}{|l|}{ To Tape } \\
\hline & $\begin{array}{c}1000 \\
500\end{array}$ & $\begin{array}{c}500 \\
3 K\end{array}$ & $\begin{array}{c}5 \\
150 K\end{array}$ & $\begin{array}{c}8000 \\
3 K\end{array}$ & & $\begin{array}{c}1.5 \\
25 \mathrm{~K}\end{array}$ & \\
\hline total events & $1200 \mathrm{M}$ & $600 \mathrm{M}$ & $40 \mathrm{M}$ & $7000 \mathrm{M}$ & & $16 \mathrm{M}$ & \\
\hline \multicolumn{8}{|l|}{ Media } \\
\hline type & gitk & $8 \mathrm{~mm}$ & $8 \mathrm{~mm}$ & $8 \mathrm{~mm}$ & $8 \sin$ & 3480 & 8480 \\
\hline number & $4 K$ & $1 \mathrm{~K}$ & $3 \mathbf{K}$ & $10 \mathrm{~K}$ & 2816 & $5.5 \mathrm{~K}$ & $10 \mathrm{n}$ \\
\hline totaf bytes $[\mathrm{Tb}]$ & 0.6 & 2 & 6 & 20 & & 1.1 & Kx: \\
\hline \multicolumn{8}{|l|}{ Reconstruction } \\
\hline MIP.sec/event & 0.1 & 15 & 300 & 6 & (r) & 15 & (x) \\
\hline Instructions/oyle & 200 & 2000 & 3000 & 2000 & 2000 & 140 & 40 \\
\hline Total [MIP-years] & 4 & 320 & 375 & 1300 & 2500 & 10 & ) 20 \\
\hline Physics & Expt. & Spokesman & & & & & \\
\hline Hyperon Radiative Decays & E761 & Vorobyov & & & & & \\
\hline $\begin{array}{l}\text { Photo-production of Charm } \\
\text { and Beauty }\end{array}$ & E687 & Butler & & & & & \\
\hline $\begin{array}{l}\text { Collider Detector at Fermilab } \\
\text { Pbar }+P \text { at } \sqrt{ } S=1.8 \mathrm{TeV}\end{array}$ & COF & $\begin{array}{l}\text { Shochet/ } \\
\text { Toliestrup }\end{array}$ & & & & & \\
\hline $\begin{array}{l}\text { High statistics Hadro- } \\
\text { production of Charm }\end{array}$ & E791 & Appel & & & & & \\
\hline $\begin{array}{l}\text { The SLD Detector at the SLC } \\
\text { Physics at the } Z \text { pole }\end{array}$ & SED & $\begin{array}{c}\text { Baltay } f \\
\text { Breidenbach }\end{array}$ & & & & & \\
\hline
\end{tabular}

Table 4. Typical Future Experiments

Its hard to change these estimates very much. At SLAC they will be driven almost completely by the I umonisity that the SLC can achieve. This effectively bounds the requirements from above. At Fermilab most of the data is coming from existing experiments with well known characteristics and relatively mature analysis codes. The physics goals dictate the requirement of high statistics and that in turn drives the computing load. Of course, estimates are al ways low so thing may be even worse.

\section{FUTURE DIRECTIONS}

So how are we to respond to continuing exponential growth? The first answer to this question is to plan - but not for too long! Both SLAC and Fermi lab now have in progress committees charged with making plans for the next five years or so. At the coarsest level the goals of the two groups are quite similar. The basic end result 
desired is a plan for incorporating the latest and most cost effective new technologies: workstations, RISC compute servers, UNIX, advanced networking, intelligent file serving, etc. At the next level the plans must necessarily diverge as they address the different situations at the two labs.

SLAC will likely wish to augment their present central facility with both more mainframes and at the same time begin to include compute servers, workstations and advanced networking (FDDI). Their real needs will be driven by the SLD data rate. This is highly uncertain at this time so I can't begin to guess the scope of what they really need to do. If it is only the factor of two I projected above then things are probably fairly straightforward. Their present model of computing can clearly be scaled a factor of two. If the real needs are much more than that they may need some more radical approach.

Fermilab does not share this luxury. Our present model of computing is just not capable of what we now require of it. It has served us relatively well for the past $5-7$ years but it's earned it's gold watch. So what is the new model of Fermilab computing to be? This is a question we are actively involved in answering right now so I can't just write down the solution. Some pieces are quite clear others almost completely opaque. The overall organizing principle - the picture that replaces the "pawnbroker" is in the latter category.

Two thing are very clear. The medium of choice for data recording and the output of PASS1 analysis will be $8 \mathrm{~mm}$ tape or some equivalent "cheap" recording medium. This is a decision driven strictly by economics. 40 terabytes of raw data plus a factor of 1.5 for PASS1 output (100 Tb total) would cost 5 - 10 MS just for the media if we used either 3480 or 9 track tapes. There is also the small problem of what you do with 500,000 tapes. The media cost for $8 \mathrm{~mm}$ is a factor of 10 less as is the number of individual volumes which have to be handled and stored. Jack Pfister will have significantly more to say on this subject in another talk at this conference (ref 4 ).

Likewise, the only apparent affordable compute engines for PASS1 processing are in the RISC/UNIX compute server class. These systems sell today for as low as \$500/MIP barebones and about \$1000/MIP with enough peripherals, software and maintenance to make them functional PASS1 compute engines. With PASS1 jobs running at more than 1000 instructions per byte either a few $8 \mathrm{~mm}$ tape drives on each system or a network connection to an "I/O server" system provides adequate $\mathrm{I} / 0$ capability for PASS1 work. How do organize and manage these new farms is more problematical. We have significant experience with managing ACP farms and are now in the process of adapting these techniques and software to these new systems (ref 5). 
The hard part, at the moment, looks like the PASS3's. Understanding the real requirements for $I / O$, CPU, shared data, online vs almost online vs offline data storage, etc. is a challenging undertaking. Some will argue that the whole job can be done with a "loosely coupled" system of distributed workstations/compute servers with only a network to tie them together for common file access, backup, etc. Others favor a more monolithic architecture with a large "central file server" at the center and arrays of RISC/UNIX compute servers hanging off the networks at the back end.

Neither of the two descriptions I just gave are models of computing. They give, perhaps, a flavor for the kind of issues with which we are wrestling. Trying to sort this all out makes for a lively set of meetings and discussions. I truly wish that I could report the beginnings of the answer to you here at this conference but I can't. If we can't begin to answer these questions in about six months you can visit our tomb. It will be just outside the new Feynman Computing Center at Fermilab and it will be constructed of unanalysed data tapes! Khruschev's old admonition of the sixties has taken on a new meaning ("We will bury you").

\section{REFERENCES}

1. "Future Computing Needs for Fermilab" Fermilab TM-1230 0062.000 December 1983 (unpubl ished)

2. "The ACP Multiprocessor System at Fermilab",I. Gaines, et.al, Comput.Phys.Commun 45,323(1987)

3. "Software for the ACP Multiprocessor System", J.Biel, et.al, Comput.Phys. Commun 45,331(1987)

4. "Recent Experiences and Future Expectations in Data Storage Technology", Jack Pfister, elsewhere in these proceedings.

5. "The Cooperative Processes Software Method for Multiprocessing Computing", C. Kaliher's, elsewhere in these proceedings. 